\title{
Influencia de la hipoxia sobre el metabolismo óseo. Rol central del factor inducible por hipoxia
}

Influence of hypoxia on bone metabolism. Central role of hipoxia-inducible factor

\author{
Elías Ernesto Aguirre Siancas \\ Docente, Facultad de Odontologia, Universidad Nacional Mayor de San Marcos. \\ Docente, Facultad de Estomatologia, Universidad Cientifica del Sur. \\ Magíster en Fisiología.
}

\begin{abstract}
Resumen
El conocimiento de la influencia de la hipoxia sobre la fisiología humana dio un salto cualitativo cuando se descubrió al factor inducible por hipoxia (HIF). Las vías moleculares que este factor de transcripción regula son variadas y complejas, cuya comprensión continuamente se sigue profundizando. En la fisiología y en la fisiopatología del tejido óseo se desarrollan varios procesos característicos, como la osificación, la remodelación y la reparación. La disminución en la disposición de oxígeno determina la estabilización del HIF en muchos grupos celulares, entre los cuales destacan las células del linaje osteogénico. Este factor regula la expresión de múltiples genes, siendo, quizás, el más importante el que codifica al factor de crecimiento vascular del endotelio (VEGF), debido a su decisiva implicancia en el proceso de osteogénesis, directamente activando a las células osteoblásticas o indirectamente mediante su potente acción angiogénica. En este artículo, se hace una revisión actualizada sobre el rol central del HIF sobre la fisiología ósea, detallando la regulación de las principales vías moleculares dependientes de dicho factor de transcripción.
\end{abstract}

Palabras clave: Hipoxia, factor inducible por hipoxia - HIF, factor de crecimiento vascular del endotelio - VEGF, angiogénesis, osteogénesis.

\begin{abstract}
Knowledge of hypoxia influencing human physiology determined discovery of hypoxia-inducible factor (HIF). Regulation of molecules by this transcription factor is varied and complex and understanding continues on. Several processes like ossification, remodeling and repair occur in bone tissue physiology and pathophysiology. Decrease in available oxygen determines HIF stabilization in many cell groups, standing out osteogenic lineage cells. This factor regulates expression of multiple genes, the most important perhaps the vascular endothelial growth factor (VEGF) due to its implication in the osteogenic process, directly activating osteoblastic cells or indirectly through potent angiogenesis. In this article, we review HIF central role on bone physiology, detailing regulation of key molecular pathways dependent of this transcription factor.
\end{abstract}

Keywords: Hypoxia, hypoxia-inducible factor - HIF, vascular endothelial growth factor - VEGF, angiogenesis, osteogenesis.

An Fac med. 2013;74(4):321-5

\section{INTRODUCCIÓN}

El tejido óseo está constituido por un conjunto de células y una matriz extracelular calcificada. Dichos componentes están en continua dinámica metabólica, metabolismo regulado por diversas condiciones entre la que destaca la variación en la disposición de oxigeno tisular ${ }^{(1,2)}$.

La hipoxia es una condición fisiológica o fisiopatológica que acompaña al tejido óseo en varios momentos de su desarrollo. En la histogénesis inicial, la osificación endocondral se caracteriza por una gradiente baja de oxígeno; esto determina la hipertrofia del condrocito previa a la aparición de las primeras trabéculas de reemplazo ${ }^{(2,3)}$.

Cuando ocurre una lesión ósea, como en una fractura o en una osteotomía, la tensión de oxígeno tisular disminuye; los mecanismos de formación aumentan, dado a que el tejido lesionado requiere una recuperación eficaz. La hipoxia es una condición de este microambiente que induce la aparición de moléculas que median los mecanismos reparativos ${ }^{(4,5)}$.

En la actualidad, muchos estudios evalúan los componentes del tejido óseo bajo hipoxia, comparándolos con controles bajo normoxia, hallándose controversias. El presente artículo re- visa la influencia de la hipoxia sobre el metabolismo óseo.

\section{EL TEJIDO ÓSEO}

El tejido óseo es un tipo especializado de tejido conectivo; brinda soporte estático y protección de órganos vitales, actúa como depósito de calcio y fosfato y contribuye a la regulación del equilibrio ácido-base. Se caracteriza porque sus células maduras están rodeadas por una matriz extracelular calcificada. La arquitectura ósea está constituida por una lámina cortical externa y un hueso trabecular interno, donde tienen su reservorio factores de crecimiento 
y mediadores que regulan su metabolismo activo. El componente mineral (cristales de hidroxiapatita) representa el $65 \%$ de su peso, mientras el agua y los componentes orgánicos, representados, sobre todo, por el colágeno tipo I, conforman el resto. Otras sustancias orgánicas presentes en la matriz son proteoglicanos, glicoproteínas, sialoproteínas y lípidos, existiendo proteínas no colágenas como la osteocalcina que interviene en su calcificación ${ }^{(1,3,6)}$.

\section{COMPONENTES CELULARES}

Existe un conjunto de células que regulan la composición de dicha matriz. Las células osteoprogenitoras -las células madres del hueso- se diferencian en osteoblastos y luego en osteocitos. Los osteoblastos son las células formadoras de hueso, y cuando plenamente activos presentan abundante retículo endoplasmático rugoso (RER) y aparato de Golgi, que refleja su función sintetizadora y secretora. Algunos osteoblastos son rodeados por la matriz ósea (osteoide) que empieza a mineralizarse, quedando encerrados en pequeñas cavidades (osteoplasto). Cuando esto ocurre, la célula se denomina osteocito. Los osteocitos son las células maduras del tejido; pueden comunicarse entre sí a través de largas prolongaciones citoplasmáticas situadas en estrechos canales denominados canalículos calcóforos. Los osteocitos reciben nutrientes suficientes para sobrevivir y así mantener el adecuado metabolismo del hueso. Además, regulan la homeostasis del calcio mediante un proceso denominado osteólisis osteocítica. Los osteoclastos son células grandes multinucleadas que derivan de los monocitos. Se hallan adheridos a la superficie del hueso en zonas de resorción ósea activa, a menudo en depresiones que ellos mismos han excavado: las lagunas de Howship ${ }^{(1,7)}$.

\section{REMODELACIÓN ÓSEA}

Normalmente el tejido óseo está en equilibrio. La formación dada por los osteoblastos es contrapesada por la resorción dada por los osteoclastos. A dicho proceso se le denomina remodelación ósea y es continua durante toda la vida. El hueso es primero resorbido por los osteoclastos y luego formado en el mismo lugar por los osteoblastos. Estas células forman la unidad metabólica básica (BMU). La remodelación ajusta el tamaño, la forma y la distribución de la masa de los huesos. Hace posible el recambio del hueso antiguo por hueso nuevo, evitando así que este tejido se fracture fácilmente. Además, la remodelación permite ajustar el tamaño de los huesos de acuerdo al esfuerzo al cual son sometidos ${ }^{(1,6,8,9)}$.

Las etapas de la remodelación tienen diferente predominio. Cuando domina la formación del hueso sobre la resorción, la masa ósea se incrementa, como ocurre en las etapas de crecimiento. En el adulto, a partir de los 35 a 40 años, la formación ósea disminuye y se impone la resorción, lo que provoca una pérdida gradual de la masa ósea ${ }^{(7,9)}$.

\section{LA TENSIÓN DE OXÍGENO EN LOS TEJIDOS CORPORALES}

Existen marcadas diferencias entre la tensión de oxígeno en los diferentes tejidos del cuerpo y la que se encuentra en el aire: $157 \mathrm{mmHg}$ a nivel mar con $760 \mathrm{mmHg}$ de presión atmosférica. La presión parcial de oxígeno $\left(\mathrm{pO}_{2}\right)$ del aire inspirado progresivamente decrece después de entrar por los pulmones y distribuirse a los diferentes tejidos. Los órganos y tejidos viven en un ambiente con tensión de oxígeno $\left(\mathrm{PO}_{2}\right)$ aproximadamente entre $2 \%$ y $9 \%$ (14 a 65 $\mathrm{mmHg}$ ), siendo el rango normal de la $\mathrm{pO}_{2}$ en la medula ósea entre $2 \%$ y $6 \%$ ( 14 a $45,6 \mathrm{mmHg}$ ) y en las zonas de reparación ósea puede llegar a 0,7\% (5,3 $\mathrm{mmHg})^{(10,11)}$.

La tensión de oxígeno en el aire ambiental se la considera típicamente como normoxia, como un estándar convencional cuando se trabaja con cultivos celulares; sin embargo, la concentración de oxígeno entre $2 \%$ y $9 \%$ ha sido recientemente apreciada por algunos investigadores como normoxia fisiológica ${ }^{(11,12)}$.

\section{ROL DEL OXÍGENO Y RESPUESTA FISIOLÓGICA A LA HIPOXIA}

El oxígeno es el sustrato para la producción de energía celular y es un regulador rápido del metabolismo. Recientes estudios han resaltado que el oxígeno tiene implicancias en el control de la proliferación celular, biotransformación y la morfogénesis durante el desarrollo de muchos tejidos, incluido el esquelético ${ }^{(13)}$.

El oxígeno está involucrado en múltiples procesos importantes en las células. Primero, el oxígeno determina el metabolismo aeróbico. Segundo, el oxígeno es necesario para activar muchas enzimas, como hidroxilasas, oxigenasas y cicloxigenasas. Tercero, la reducción de oxígeno en los tejidos interfiere en la síntesis de colágeno, porque el oxígeno es requerido para la hidroxilación de la lisina y la prolina. Cuarto, el oxígeno es un importante señalizador molecular, regulando la expresión de varios genes entre los que destaca el factor inducible por hipoxia (HIF). Además, el oxígeno ha estado implicado en el mantenimiento de las células madre, su movilización y su reclutamiento en los sitios de lesión ${ }^{(14)}$.

La respuesta a la hipoxia puede ser esquematizada en tres sistemas: a) de detección o sensor de oxígeno, b) de regulación, controlando la expresión de una amplia serie de genes, y c) efector múltiple, que incluye no solo expresión génica, sino múltiples cambios funcionales que van desde la estimulación de moléculas vasodilatadoras hasta las variaciones en la afinidad hemoglobinaoxígeno. Existe hipoxia transitoria durante el ejercicio, en la apnea del sueño, en la sepsis o en tejidos traumatizados, en la hipoxia crónica en la altura o en zonas tisulares menos oxigenadas, como ocurre en el cáncer. En los mamíferos, la respuesta a la hipoxia es compleja, e incluye: adaptaciones de la función res- 
piratoria y hemodinámica, del metabolismo intermedio y de la función renal. Estas compensaciones se manifiestan, entre otras, en cambios hormonales, de mediadores y de actividad enzimática, e implican variaciones en la expresión de una serie de moléculas ${ }^{(15,16)}$.

\section{LA HIPOXIA, SU RELACIÓN CON ALGUNAS PATOLOGÍAS HUMANAS}

En condiciones fisiológicas, las especies reactivas de oxígeno (ROS) son generadas por varios compartimientos subcelulares -las mitocondrias, la membrana celular, los lisosomas, los peroxisomas y el retículo endoplasmático-, siendo inactivadas por la cascada de la superóxido dismutasa. En los ciclos de hipoxia y reoxigenación encontrados en las personas que padecen de apnea obstructiva del sueño (AOS), se produce una excesiva cantidad de radicales libres, alterándose esta neutralización oxidativa, de una manera similar a la observada en las lesiones isquémicas, como en la insuficiencia cardiaca y en el accidente cerebrovascular ${ }^{(17)}$.

La AOS es debida al colapso repetitivo de la vía aérea superior durante el sueño. Estudios epidemiológicos a gran escala han demostrado que la AOS no tratada es un factor independiente de riesgo cardiovascular, como lo es la hipertensión. En estudios realizados en ratones a los que se sometió a hipoxia intermitente crónica durante el sueño (diseño simulando a la AOS) se encontró un aumento en la apoptosis de las estructura de la parte media del lóbulo temporal, implicado en el aprendizaje y la memoria. Además, se evidenció la aparición de una serie de vías que se desencadenan en respuesta a la hipoxia intermitente, como la excitotoxicidad del glutamato, el estrés oxidativo, la disfunción mitocondrial, la regulación positiva de mediadores pro-inflamatorios y la regulación alterada de las vías apoptóticas, siendo los más afectados el aparato cardiocirculatorio y el sistema nervioso ${ }^{(17,18)}$.
En el cáncer, el ambiente hipóxico es una característica importante, estableciéndose por defectos en la vascularización y producto del intenso metabolismo neoplásico. Al igual que en los procesos fisiológicos, el factor de crecimiento vascular del endotelio (VEGF) es el factor responsable de la neovascularización de un tejido (angiogénesis); pero en estas circunstancias determina un círculo vicioso patológico de actividad angiogénica aberrante, contribuyendo al crecimiento tumoral. Adicionalmente, la secreción del VEGF por las células tumorales actúa como un inmunosupresor de citoquinas, disminuyendo de manera directa la respuesta inmune antitumoral ${ }^{(19,20)}$.

\section{ROL BENÉFICO DE LA HIPOXIA SOBRE EL METABOLISMO ÓSEO}

El acoplamiento angiogénesis-osteogénesis es vital para el inicio, mantenimiento y reparación del tejido óseo. La hipoxia es la mayor fuerza estimuladora de este acoplamiento, regulando su programa genético ${ }^{(3,21)}$.

La angiogénesis es una condición necesaria durante el desarrollo del hueso, tanto prenatal como posnatal. Si se inhibe la angiogénesis, el resultado es la persistencia del tejido óseo en su etapa fibrosa en lugar de proseguir hacia su etapa mineralizada. En el proceso de osificación endocondral, las células mesenquimales adyacentes a la zona de cartílago hipertrófico viven en un ambiente hipóxico. Estas células del mesénquima empiezan a expresar niveles crecientes de VEGF, que dirige la formación de los vasos sanguíneos que invadirán a los condrocitos hipertróficos, los cuales también empiezan a sufrir procesos de apoptosis, evidenciándose el adecuado reemplazo del cartílago por las trabéculas óseas ${ }^{(3,21,22)}$.

En los procesos de injuria sobre el tejido óseo - como una fractura o una osteotomía- se produce una interrupción vascular que crea un gradiente hipóxico en la zona de lesión, en donde la tensión de oxígeno en el centro de la herida es más baja. In vivo, este microambiente hipóxico estimula la expresión de una variedad de moléculas sintetizadas por las células inflamatorias, los fibroblastos, las células endoteliales y los osteoblastos ${ }^{(15,23)}$.

Muchas familias de moléculas que actúan como factores locales y sistémicos median la angiogénesis y la osteogénesis, siendo los principales el factor de crecimiento de fibroblastos (FGF), factor de crecimiento transformante (TGF), factor de crecimiento similar a la insulina (IGF), factor de crecimiento derivado de plaquetas (PDGF) y factor de crecimiento vascular del endotelial (VEGF). Además, el acoplamiento de la osteogénesis y la angiogénesis puede ser regulado por factores sistémicos, tales como la hormona paratiroidea (PTH) y la hormona de crecimiento (GH) ${ }^{(3,24,25)}$.

- Factor de crecimiento vascular del endotelio (VEFG).- Su presencia en el hematoma, después de la lesión ósea inicial, activa la angiogénesis y modula la adecuada mineralización. El VEFG regula el reclutamiento, la supervivencia y la actividad de las células endoteliales, osteoblastos y osteoclastos. Además, el VEGF aumenta la proliferación y diferenciación osteoblástica ${ }^{(3)}$.

- Factor de crecimiento de fibroblastos (FGF).- Regula la proliferación, la migración y la diferenciación de las células implicadas en la reparación ósea. La mayoría de los estudios se ha centrado en FGF-2, encontrándose que este factor estimula la formación ósea ${ }^{(3)}$.

- Factor de crecimiento transformante (TGF).- Los miembros de la familia del factor de crecimiento transformante (TGF) incluyen principalmente al TGF $\beta$ y a las proteínas morfogenéticas óseas BMP-2 y BMP-7. Estos factores estimulan la proliferación y la diferenciación de células osteoprogenitoras y mesenquimales, y promueven la angiogénesis y simultáneamente la repara- 
ción ósea. El BMP-2 y 7 tienen una acción directa osteogénica y angiogénica y el BMP-7 mejora sinérgicamente la actividad angiogénica del FGF-2 y del TGT $\beta^{(3)}$.

\section{EJE HIPOXIA - ANGIOGÉNESIS - OSTEOGÉNESIS: ROL CENTRAL DEL FACTOR INDUCIBLE POR HIPOXIA (HIF)}

El HIF es un complejo proteico heterodimérico que está compuesto por dos subunidades que se expresan constitutivamente: la subunidad HIF-1 $\beta$, de ubicación nuclear, y la subunidad HIF- $\alpha$, de ubicación citosólica. La subunidad HIF- $\alpha$ se presenta como HIF- $1 \alpha$, HIF$2 \alpha$ y HIF- $3 \alpha$, siendo la HIF- $1 \alpha$ la primera descrita y que ha sido investigada más ampliamente. Esta HIF- $\alpha$ es sensible a la presencia de oxígeno tisular, siendo degradada constantemente. La HIF es una molécula que regula la homeostasis del oxígeno y tiene rol importante en el desarrollo, en la fisiología y en la patología ${ }^{(12,15,26)}$.

Este complejo proteico es sintetizado por múltiples células del organismo. Las subunidades HIF- $\alpha$ y HIF- $1 \beta$ presentan pesos moleculares entre 120 y $130 \mathrm{kD}$ y 91 y $94 \mathrm{kD}$, respectivamente. El HIF es un factor de transcripción del tipo hélice-lazo-hélice básico (bHLH). Regula la transcripción de múltiples genes -como el que codifica al factor de crecimiento derivado de las plaquetas, a la eritropoyetina y a la transferrina- siendo, quizás, el más importante el gen que codifica al factor de crecimiento vascular del endotelio (VEGF), debido a su decisiva implicancia en el proceso de osteogénesis, directamente activando a las células osteoblásticas o indirectamente mediante su potente acción angiogénica. Diversas células del organismo, entre las que están los osteoblastos, regulan la presencia del HIF mediante un sistema enzimático: la propil (tipo PHD-2) y la asparagil (tipo FIT-1) hidroxilasas. En normoxia tisular, la HIF- $\alpha$ sufre hidroxilación por la PHD-2 y por la FIT-1; luego, la proteína de von Hippel-Lindau (pVHL) reconoce a este complejo hidroxilado y lo marca para su degradación por el proteosoma. La PHD-2 y la FIT-1 requieren oxígeno para cumplir sus funciones. Por ello, en condiciones de normoxia la HIF- $\alpha$ es continuamente degradada. Pero, cuando la disposición tisular disminuye (hipoxia), las enzimas se inactivan y ya no reaccionan sobre la subunidad HIF- $\alpha$ y esta empieza a acumularse rápidamente en el cito$\operatorname{sol}^{(3,15,26)}$.

La unidad HIF- $\alpha$, como se ha descrito, está regulada por un mecanismo enzimático sensible a oxígeno; en contraste, la HIF-1 $\beta$ se expresa estable e independiente de la disposición de oxígeno tisular. Cuando disminuye la tensión de oxígeno (niveles de concentración menores a 5\%), la proteína HIF- $\alpha$ se estabiliza y se desplaza al núcleo, acoplándose (heterodimerizándose) con la HIF-1 $\beta$. Este complejo molecular recién formado empieza a regular la transcripción génica ${ }^{(15,27,28)}$.

En múltiples estudios se ha investigado el efecto de diferentes concentraciones de oxígeno sobre la estabilización y la expresión del HIF y la transcripción de su principal objetivo, el VEGF.

Se ha evaluado células obtenidas del periostio de la calota de ratas SpragueDawley. Estas fueron cultivadas y sometidas, un grupo, a hipoxia $\left(5 \% \mathrm{de}_{2}\right)$ y, el otro grupo, a normoxia ( $20 \%$ de $\mathrm{O}_{2}$ ). Se estudió la expresión de ARNm y de la síntesis proteica de HIF y de VEGF, entre otras moléculas. Se hizo controles al 1으, 2o $3^{\circ}$ y y $4^{\circ}$ días para evaluar la expresión de ARMm, siendo en los cuatro controles mayores la presencia de HIF y VEGF en hipoxia comparado con normoxia. Sobre el 4o día se determinó la síntesis proteica e igualmente esta fue mayor en hipoxia comparado con normoxia ${ }^{(29)}$.

Al estudiar los efectos de la hipoxia (simulando oxígeno ambiental a 3850 msnm) en un grupo de personas en actividad física (bicicleta estacionaria) comparada con un grupo a nivel del mar, se halló incremento en los niveles de HIF-1 y de VEGF en el grupo de per- sonas que realizaron el ejercicio en el ambiente simulado a $3850 \mathrm{msnm}^{\left({ }^{(30}\right)}$.

Se ha investigado la expresión de HIF-1 $\alpha$ y de VEGF en células madres mesenquimales de ratones. Las células fueron incubadas en una cámara bajo hipoxia $\left(2 \%\right.$ y al $5 \%$ de $\mathrm{O}_{2}$ ) y otro grupo celular bajo normoxia $\left(21 \%\right.$ de $\left.\mathrm{O}_{2}\right)$, durante 6 a 24 horas. Se encontró que los niveles de la HIF-1 $\alpha$ y de VEGF estaban aumentados en los cultivos a 5\% de $\mathrm{O}_{2}$, pero se inhibieron a $2 \%$ de $\mathrm{O}_{2}$, al igual que en normoxia ${ }^{(31)}$.

En otro estudio, se comparó las células madre mesenquimales obtenidas de médula ósea (MSCs) de la cresta iliaca por medio de una biopsia por aspiración de donantes sanos de 19 a 32 años de edad. Se dividió las células en tres grupos: uno expuesto a normoxia y dos expuestos a hipoxia $\left(2 \% \mathrm{de}_{2}\right)$, en uno de cuales las células fueron manipuladas genéticamente para que sobreexpresen HIF (grupo HIF-MSCs). Se encontró que los niveles de expresión de VEGF y de c-Met (un gen que codifica un receptor para el factor de crecimiento de hepatocitos; dicho factor interviene en las etapas iniciales de la angiogénesis en varios tejidos) fueron mayores en el grupo HIF-MSCs sobre el grupo expuesto a hipoxia pero no manipulado genéticamente; a su vez, este grupo expresó mayor VEGF y c-Met que el expuesto a normoxia ${ }^{(32)}$.

\section{CONCLUSIONES}

En la fisiología humana, la mayoría de sistemas regula su equilibrio (homeostasis) mediante la retroalimentación negativa, el mecanismo de regulación por excelencia, en el cual el aumento o la disminución de una condición fisiológica o fisiopatológica es controlada por la activación de los sistemas reguladores que tienden a disminuir o a aumentar dicha condición, respectivamente, llevando los valores alterados hacia el nivel de referencia basal. Así se regula, por ejemplo, la presión arterial, la temperatura corporal y la glicemia. La disminución del oxígeno 
tisular disminuye el metabolismo aeróbico y las diversas reacciones mediadas por el oxígeno descritas en el presente artículo. La estabilización del HIF, en un medio tisular hipóxico, tiene como objetivo restablecer la vía aeróbica en la obtención de energía, además de los mecanismos moleculares que se desarrollan en las células ante la adecuada oxigenación; la transcripción del gen de VEGF por el HIF permite el retorno del oxígeno al tejido hipóxico mediante su potente acción angiogénica.

La hipoxia tisular, sobre todo por debajo de la concentración de 5\% de $\mathrm{O}_{2}$, permite la estabilización de la HIF- $\alpha$ citosólica que heterodimeriza con la HIF- $\beta$ nuclear para formar el HIF, el cual determina la expresión de múltiples genes que median diversas vías moleculares. Estas vías permiten a las células primero su adaptación a la hipoxia y segundo su diferenciación y proliferación, con la consecuente síntesis de diversas moléculas de señalización y factores de transcripción, que promueven una cascada de eventos moleculares que aceleran el metabolismo de las células que intervienen en la fisiología del tejido óseo.

\section{REFERENCIAS BIBLIOGRÁFICAS}

1. Gartner L, Hiatt J. Histologia Básica. Barcelona: Elsevier Saunders. 2011:84-9.

2. D'Ippolito G, Diabira S, Howard G, Roos B, Schiller $P$. Low oxygen tension inhibits osteogenic differentiation and enhances stemness of human MIAMI cells. Bone. 2006;39(3):513-22.

3. Mamalis AA, Cochran DL. The therapeutic potential of oxygen tension manipulation via hypoxia inducible factors and mimicking agents in guided bone regeneration. Arch Oral Biol. 2011;56(12):1466-75.

4. Wu E, Li H, Zhao T, Fan J, Ma X, Xiong L, et al. Effect of hypoxia on the gene profile of human bone marrow-derived mesenchymal stem cells. 2007;59(2):227-32.

5. Towler D. Vascular biology and bone formation. $J$ Clin Invest. 2007;117(6):1477-80.
6. Dvorkin M, Cardinali D, lermoli R. Best \& Taylor: Bases Fisiológicas de la Práctica Médica. $14^{\text {ta }}$ ed Buenos Aires: Editorial Médica Panamericana. 2010:783-90.

7. Gómez de Ferraris M, Campos Muñoz A. Embriología, Histología e Ingenieria Tisular Bucodental. $3^{a}$ ed. México DF: Editorial Médica Panamericana. 2009:381-7.

8. Bilezikian J, Raisz L, Martin T. Principles of Bone Biology. Vol. 1. $3^{\text {a }}$ ed. New York: Academic Press. 2008:5-6.

9. Michael J, Sirca S. Fisiología Humana. México DF: Editorial El Manual Moderno. 2012:494-6.

10. Villarruel S, Boehm C, Pennington M, Bryan J, Powel K, Muschlen G. The effect of oxygen tension on the in vitro assay of human osteoblastic connective tissue progenitor cells. J Orthop Res. 2008;26(10):1390-7

11. Mohyeldin A, Garzón T, Quiñones A. Oxygen in stem cell biology: a critical component of the stem cell niche. Cell Stem Cell. 2010;7(2):150-61.

12. Holzwarth C, Vaegler M, Gieseke F, Pfister S, Handgretinger R, Kerst G, et al. Low physiologic oxygen tensions reduce proliferation and differentiation of human multipotent mesenchymal stromal cells. BMC Cell Biol. 2010;11:11.

13. Yang DC, Yang MH, Tsai CC, Huang TF, Chen $\mathrm{YH}$ Hung SC. Hypoxia inhibits osteogenesis in human mesenchymal stem cells through direct regulation of RUNX2 by TWIST. PLoS One. 2011;6(9):e23965.

14. Lu CH, Saless N, Wang X, Sinha A, Decker S, Kazakia $\mathrm{G}$, et al. The role of oxygen during fracture healing. Bone. 2013;52(1):220-9.

15. Semenza GL. Regulation of oxygen homeostasis by hypoxia-inducible factor 1. Physiology. 2009;24:97106.

16. Tang CH, Lu DY, Tan TW, Fu WM. Yang RS. Ultrasound induces hypoxia-inducible factor-1 activation and inducible nitric oxide synthase expression through Integrin/integrin-linked kinase/Akt/mammalian target of rapamycin pathway in osteoblasts. J Biol Chem. 2007;282(35):25406-15.

17. Wang Y, Zhang S, Gozal D. Reactive oxygen species and the brain in sleep apnea. Respir Physio Neurobiol. 2010;174(3):307-16.

18. Dumitrascu R, Heitmann J, Seeger W, Weissmann N Schulz R. Obstructive sleep apnea, oxidative stress and cardiovascular disease: lessons from anima studies. Oxid Med Cell Longev. 2013;2013:234631. doi: 10.1155/2013/234631.

19. Collet G, Skrzypek K, Grillon C, Matejuk A, El HafniRahbi B, Lamerant-Fayel N, et al. Hypoxia control to normalize pathologic angiogenesis: potential role for endothelial precursor cells and miRNAs regulation. Vascul Pharmacol. 2012;56(5-6):252-61.

20. Asis Palazón, Julián Aragonés, Aizea MoralesKastresana, Ortiz de Landazuri M, Melero I. Molecular pathways: hypoxia response in immune cells fighting or promoting cancer. Clin Cancer Res. 2012;18:1207-13.

21. Araldi E, Schipani E. Hypoxia, HIFs and bone development. Bone. 2010;47(2):190-6.
22. Leijten J, Moreira L, Landman E, van Blitterswijk C, Karperien M. Hypoxia inhibits hypertrophic differentiation and endochondral ossification in explanted tibiae. PLoS One. 2012;7(11):e49896.

23. Kolar P, Gaber T, Perka C, Duda G, Buttgereit F. Human early fracture hematoma is characterized by inflammation and hypoxia. Clin Orthop Relat Res. 2011;469(11):3118-26.

24. Schmidt-Bleek K, Schell H, Schulz N, Paula Hoff $P$, Perka C, Buttgereit F, et al. Inflammatory phase of bone healing initiates the regenerative healing cascade. Cell Tissue Res. 2012;347(3):567-73.

25. Wang Y, Li J, Wang Y, Lei L, Jiang Ch, An S, et al. Effects of hypoxia on osteogenic differentiation of rat bone marrow mesenchymal stem cells. Mol Cell Biochem. 2012;362(1-2):25-33.

26. Wan C, Shao J, Gilbert SR, Riddle RC, Long F, Johnson RS, et al. Role of HIF-1alpha in skeletal development. Ann N Y Acad Sci. 2010;1192:322-6.

27. Bracken C, Fedele A, Linke S, Balrak W, Lissy K, Whitelaw M, et al. Cell-specific regulation of hypoxiainducible factor (HIF)-1 $\alpha$ and HIF-2 $\alpha$ stabilization and transactivation in a graded oxygen environment. J Biol Chem. 2006;281(32):22575-85

28. Wang Y, Wan C, Deng L, Liu X, Cao X, Gilbert S, et al. The hypoxia-inducible factor a pathway couples angiogenesis to osteogenesis during skeletal development. J Clin Invest. 2007;117(6):1616-26.

29. Ichijima T, Matsuzaka K, Tonogi M, Yamane GY, Inoue T. Osteogenic differences in cultured rat periosteal cells under hypoxic and normal conditions. Exp Ther Med. 2012;3(2):165-70.

30. Vogt, M, Puntschart A, Geiser J, Zuleger C, Billeter $\mathrm{R}$, Hoppeler M. Molecular adaptations in human skeletal muscle to endurance training under simulated hypoxic conditions. J Appl Physiol. 2001;91(1):17382.

31. Ishizuka T, Hinata T, Watanabe Y. Superoxide induced by a high-glucose concentration attenuates production of angiogenic growth factors in hypoxic mouse mesenchymal stem cells. J Endocrinol. 2011;208(2):147-59.

32. Razban V, Sahebqadam A, Khoshdel A. HIF-1 $\alpha$ overexpression induce angiogenesis in mesenchymal stem cells. Biores Open Access. 2012;1(4):17483.

Artículo recibido el 9 de junio de 2013 y aceptado para publicación el 30 de junio de 2013.

Conflicto de intereses:

No existen en el presente trabajo.

Correspondencia:

Elías Ernesto Aguirre Siancas

Dirección: Jr. Pomacanchi 326. Lima 30

Celular: 9979-38372

Correo electrónico: elias_aguirre@yahoo.com 